\title{
A Clinical Comparison of Home-Based and Hospital-Based Exercise Programs Following Arthroscopic Capsulolabral Repair for Anterior Shoulder Instability
}

\author{
Illker Eren, Nazan Canbulat, Ata Can Atalar, Şule Meral Eren, Ayla Uçak, \\ Önder Çerezci, and Mehmet Demirhan
}

\begin{abstract}
Context: Ideal rehabilitation method following arthroscopic capsulolabral repair surgery for anterior shoulder instability has not been proven yet. Although rapid or slow protocols were compared previously, home- or hospital-based protocols were not questioned before. Objective: The aim of this prospective unrandomized controlled clinical trial is to compare the clinical outcomes of home-based and hospital-based rehabilitation programs following arthroscopic Bankart repair. Design: Nonrandomized controlled trial. Setting: Orthopedics and physical therapy units of a single institution. Patients: Fifty-four patients (49 males and 5 females) with an average age of 30.5 (9.1) years, who underwent arthroscopic capsulolabral repair and met the inclusion criteria, with at least 1-year follow-up were allocated into 2 groups: home-based $(n=33)$ and hospital-based $(n=21)$ groups. Interventions: Both groups received identical rehabilitation programs. Patients in the home-based group were called for follow-up every 3 weeks. Patients in the hospital-based group admitted for therapy every other day for a total of 6 to 8 weeks. Both groups were followed identically after the eighth week and the rehabilitation program continued for 6 months. Main Outcome Measures: Clinical outcomes were assessed using Disabilities of Arm Shoulder Hand, Constant, and Rowe scores. Mann-Whitney $U$ test was used to compare the results in both groups. Wilcoxon test was used for determining the progress in each group. Results: Groups were age and gender matched $(P=.61, P=.69)$. Average number of treatment sessions was 13.8 (7.3) for patients in the hospital-based group. Preoperative Disabilities of Arm Shoulder Hand (27.46 [11.81] vs 32.53 [16.42], $P=.22$ ), Constant (58.23 [14.23] vs 54.17 [10.46], $P=.13$ ), and Rowe (51.72 [15.36] vs 43.81 [19.16], $P=.12$ ) scores were similar between groups. Postoperative scores at sixth month were significantly improved in each group $(P=.001, P=.001$, and $P=.001)$. No significant difference was observed between 2 groups regarding clinical scores in any time point. Conclusions: We have, therefore, concluded that a controlled home-based exercise program is as effective as hospital-based rehabilitation following arthroscopic capsulolabral repair for anterior shoulder instability.
\end{abstract}

Keywords: Bankart repair, shoulder rehabilitation, home-based rehabilitation, hospital-based rehabilitation

Arthroscopic repair has become the primary surgical option for the treatment of recurrent anterior shoulder instability, as the studies reported comparable outcomes between open and arthroscopic techniques. ${ }^{1-3}$ Advances in surgical techniques and implant quality provided more secure repair of the capsulolabral tissue to glenoid rim and allowed earlier rehabilitation programs to gain range of motion (ROM). ${ }^{4}$ Reported failures of arthroscopic repairs are thought to be related to technical factors and failure to recognize other accompanying pathologies responsible for glenohumeral instability. ${ }^{5}$ Successful operative outcome in patients with shoulder instability depends on accurate preoperative evaluation, careful diagnostic arthroscopy to evaluate concomitant pathologies, proper arthroscopic repair technique, and effective postoperative rehabilitation program. The delicate balance between stability and mobility, as well as the complex interplay between the static and the

Eren and Demirhan are with the Department of Orthopaedics and Traumatology, School of Medicine, Koc University, Istanbul, Turkey. Eren is also with the Koc Universitesi Hastanesi, Istanbul, Turkey. Canbulat is with the Department of Physical Therapy and Rehabilitation, School of Medicine, Koc University, Istanbul, Turkey. Atalar is with the Department of Orthopaedics and Traumatology, Acibadem University School of Medicine, Istanbul, Turkey. Eren, Uçak, and Çerezci are with the Department of Physical Therapy and Rehabilitation, VKV American Hospital, Istanbul, Turkey. Eren (Ilker.eren@gmail.com) is corresponding author. dynamic stabilizing factors requires a meticulous rehabilitation approach. ${ }^{6}$

Home-based therapy programs are the mainstay rehabilitation strategy in many clinical conditions. They have clear advantages regarding the work force allocation, cost and management of resources. ${ }^{7}$ Published therapy protocols such as The American Society of Shoulder and Elbow Therapists' guideline on shoulder instability provide reliable and applicable data. ${ }^{8}$ On the other hand, patient adherence to home-based protocols is a common problem affecting results. ${ }^{9,10}$ It was shown that home-based protocols demonstrated the lowest levels of adherence among physical therapy modalities. ${ }^{11,12}$

Previously, aggressive rehabilitation was associated with recurrence and failure. ${ }^{13,14}$ As reported in more recent studies, improved surgical techniques and implants allowed earlier rehabilitation in selected patient groups with accelerated rehabilitation protocols. As the arthroscopic Bankart repair causes little inflammatory response in the capsule and surrounding tissues, patients are relatively pain free and home-based exercise programs are the first choice in rehabilitation. Immediate postoperative exercise programs are shown to be effective in functional recovery without harm to the repaired tissue. ${ }^{15,16}$

There is evidence on both safety and efficacy of homebased rehabilitation and necessity of supervised hospital-based approach. Considering the controversies, home- and hospital-based 
rehabilitation protocols should be compared in each clinical setting to established reliable evidence.

The purpose of this study was to compare the clinical results of patients treated with home-based program and hospital-based therapy, following arthroscopic capsulolabral repair for anterior shoulder instability. We have hypothesized that home-based patientcontrolled postoperative rehabilitation after arthroscopic repair would be as effective as a supervised hospital-based rehabilitation.

\section{Methods}

Patients who were diagnosed with recurrent anterior shoulder instability and underwent an arthroscopic capsulolabral repair were enrolled into the study. Following the surgery, patients were allocated to home-based or hospital-based groups. Functional outcomes were assessed before the surgery, at the 3rd, 6th and 12th months. During the study period, 65 patients who had arthroscopic Bankart repair were assessed for eligibility and 54 patients with an average age of 30.5 (9.1) were included (Figure 1). Thirty-three patients (29 males and 4 females) with an average age of 29.9 (8.7) were allocated to the home-based therapy group (HB group) and 21 (20 males and 1 female) patients with an average age of 29.9 (8.7) to the hospital-based supervised group (SV group). Group distributions and preoperative scores are summarized in Table 1.

Inclusion criteria were: (1) presence of a traumatic recurrent unidirectional anteroinferior shoulder instability in 1 shoulder, (2) patients aged 16 years and older with closed epiphyseal plate, (3) Bankart lesion with less than $20 \%$ bone loss, (4) minimum

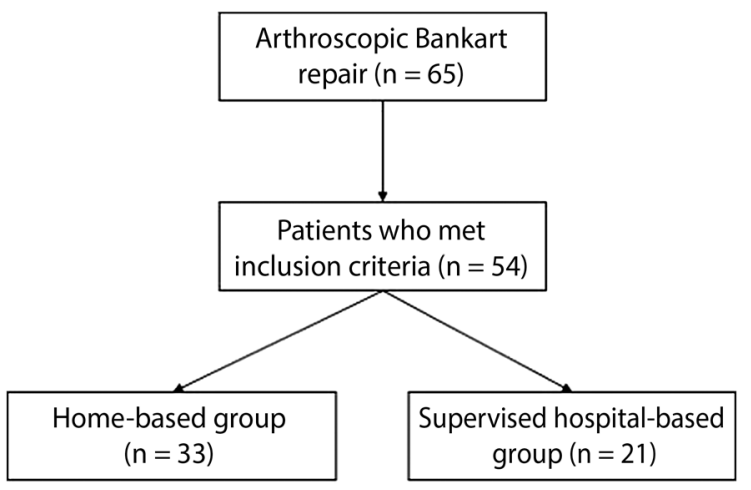

Figure 1 - Patient inclusion and exclusion information and final included sample. follow-up of 1 year, and (5) appropriate patient consent. Exclusion criteria were (1) revision instability surgery, (2) patients with glenoid defect greater than 20\%, (3) engaging Hill-Sachs' lesion requiring remplissage, (4) posterior or multidirectional shoulder instability, and (5) associated rotator cuff tear.

Allocation to the rehabilitation group was performed in a nonrandomized fashion, according to the patients' preferences. Patients were allocated into 2 groups: SV group, who received treatment in the outpatient physiotherapy unit of the hospital and HB group, who performed the structured rehabilitation program at home without supervision.

Preoperative and postoperative clinical assessments were performed using a quick version of "Disabilities of Arm Shoulder Hand" (DASH) score, Constant score and Rowe score. ${ }^{17-21}$ The DASH score is a patient-reported upper-extremity outcome questionnaire consisting of 11 questions regarding daily activities, social life, work, pain, and sleep. The Rowe score is a wellrecognized score for the postoperative assessment of labral repairs, consisting of 3 questions addressing stability (subluxation and dislocations), motion (external rotation), and function (limitation in daily activities). Constant score is a clinical completed shoulder scoring system consisting of subjective (pain and activity) and objective (range of motion and strength) subsections.

\section{Surgical Technique}

Surgeries were performed by the senior surgeon, using the identical repair technique. Patients were prepared in lateral decubitus position under traction. Following arthroscopic examination of the shoulder, ill-healed labrum was detached from the glenoid neck. Three fiberwire loaded knotless anchors (PushLock; Arthrex, Naples, FL) were used for fixation. An arm sling with cushion support for abduction was utilized postoperatively. Patients were discharged from the hospital in the morning following operation.

\section{Rehabilitation Program}

Apart from being home or hospital based, rehabilitation programs were constructed to be identical regarding the exercises (Table 2).

\section{Phase I (Maximum Protection Phase, 0-4 Wk)}

Arm sling was utilized for 4 weeks following surgeries. Table top activities (writing, eating, using computer, etc) without sling were encouraged. Postural exercises with sling and isometric exercises for deltoid strengthening were initiated on the day after surgery.

Table 1 Both Groups Were Age and Gender Matched, Number of Dislocations and Duration of Symptoms Were Similar

\begin{tabular}{|c|c|c|c|}
\hline & Home-based group $(n=33)$ & Supervised group $(n=21)$ & $P$ value $^{a}$ \\
\hline Age, $y$ & $29.9(8.7)$ & $31.5(9.6)$ & .61 \\
\hline Gender $\mathrm{M} / \mathrm{F}$ & $29 / 4$ & $20 / 1$ & .64 \\
\hline Number of dislocations & $4.9(4.5)$ & $3.0(2.0)$ & .18 \\
\hline Duration of symptoms, mo & $37.0(60)$ & $11.56(14.87)$ & .18 \\
\hline DASH & $27.46(11.81)$ & $32.53(16.42)$ & .22 \\
\hline Constant & $58.23(14.23)$ & $54.17(10.46)$ & .13 \\
\hline Rowe & $51.72(15.36)$ & $43.81(19.16)$ & .12 \\
\hline
\end{tabular}

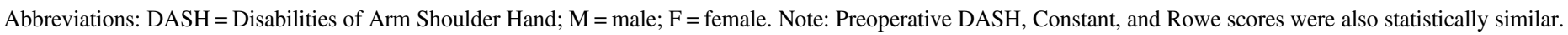

aann-Whitney $U$ test. 
Table 2 Durations, Goals, and Key Aspects of the Therapy Program in Each Phase

\begin{tabular}{|c|c|c|}
\hline Rehabilitation phases & Goals & Activities and exercises \\
\hline $\begin{array}{l}\text { I: Maximum protection } \\
\text { phase } \\
(0-4 \text { wk })\end{array}$ & $\begin{array}{l}\text { Allow healing of capsule ligament- } \\
\text { abrum complex }\end{array}$ & $\begin{array}{l}\text { Sling for } 4 \text { wk } \\
\text { Table top activities } \\
\text { Isometric deltoid strengthening } \\
\text { Postural exercises with sling } \\
\text { No shoulder ROM } \\
\text { ROM for elbow, wrist, and fingers }\end{array}$ \\
\hline $\begin{array}{l}\text { II: Limited motion phase } \\
(5-8 \mathrm{wk})\end{array}$ & $\begin{array}{l}\text { Preserve the fixation while gradually } \\
\text { increasing ROM }\end{array}$ & $\begin{array}{l}\text { Passive shoulder ROM } \\
\text { Forward flexion max } 140^{\circ} \\
\text { External rotation max } 20^{\circ} \\
\text { Active-assisted ROM with L-bar and pulley } \\
\text { Isometric exercises for IR and ER } \\
\text { Isometric scapulothoracic exercises } \\
\text { No external rotation over } 60^{\circ} \text { abduction }\end{array}$ \\
\hline $\begin{array}{l}\text { III: Medium protection phase } \\
(9-12 \mathrm{wk})\end{array}$ & Full and pain-free ROM & $\begin{array}{l}\text { Active ROM exercises } \\
\text { Full elevation } \\
\text { External rotation max } 40^{\circ} \\
\text { Isotonic flexion, ER, IR, and extension strengthening exercises } \\
\text { (dumbbell or thera band) }\end{array}$ \\
\hline $\begin{array}{l}\text { IV: Minimum protection phase } \\
(13-21 \mathrm{wk})\end{array}$ & $\begin{array}{l}\text { Improvement of neuromuscular control, } \\
\text { advancement in proprioception and } \\
\text { gaining agility }\end{array}$ & $\begin{array}{l}\text { Capsular stretching exercises for full ROM } \\
\text { Strengthening for rotator cuff and scapular stabilizers } \\
\text { (dumbbell and thera band) } \\
\text { Closed kinetic chain exercises for scapular stabilizers } \\
\text { (push-up, press-up, and rowing) } \\
\text { Plyometric exercises after 16th wk }\end{array}$ \\
\hline $\begin{array}{l}\text { V: Functional phase } \\
\text { (22nd wk and later) }\end{array}$ & Return to sport & $\begin{array}{l}\text { Functional strengthening to shoulder and scapulothoracic } \\
\text { muscles } \\
\text { Proprioceptive exercises } \\
\text { Plyometric exercises }\end{array}$ \\
\hline
\end{tabular}

Abbreviations: ER, external rotation; IR, internal rotation; ROM, range of motion.

\section{Phase II (Limited Motion Phase, 5-8 Wk)}

After the fourth week, sling support was ceased, passive and active ROM exercises were begun. At this point, group allocation was performed. Patients in home-based exercise group were instructed to fill exercise checklist daily and were called for follow-up every 3 weeks. Patients in SV group were called for therapy on every other day (3 times weekly). Follow-up was identical for both groups after the eighth week. Active assisted ROM exercises were performed in addition to isometric strengthening exercises. External rotation over $60^{\circ}$ abduction was not allowed in any of the patients.

\section{Phase III (Medium Protection Phase, 9-12 Wk)}

At the end of this period, patients were expected to gain pain-free full ROM. Active ROM exercises were performed. Isotonic strengthening exercises were initiated with dumbbell or Thera band.

\section{Phase IV (Minimum Protection Phase, 12-21 Wk)}

Strength, proprioception, and agility exercises were initiated. Capsular stretching exercises were used for full ROM when necessary. Strengthening exercises for rotator cuff and scapular stabilizers, closed kinetic chain exercises were initiated. Plyometric exercises were also initiated after the fourth month.

\section{Phase V (Functional Phase)}

Aggressive strengthening exercises and plyometric exercises was added to the rehabilitation program. Returning to sports was aimed for selected patients.
Rehabilitation program continued for 6 months. To complete each phase and proceed to the next, an agreed decision was formed between the surgical and rehabilitation teams. Functional status of the patients was assessed using DASH, Constant, and Rowe scores at 3rd, 6th, and 12th months.

Statistical analyses were performed using SPSS software (IBM, New York, NY). In addition to descriptive methods such as average and SD, Mann-Whitney $U$ test was used to compare the results in both groups, and Wilcoxon test is used to measure the progress in each group. $P$ value below .05 was considered as statistically significant in all tests.

\section{Results}

No significant difference was observed in gender distribution $(P=.64)$ and age $(P=.61)$. Fifty-two of the patients reported participation in sports at a recreational level and 2 patients at a professional level (Table 1). Average number of dislocations (HB: 4.9 [4.5], SV: 3.0 [2.0]) and duration of symptoms from the first dislocation (HB: 37.0 [60] mo, SV: 11.56 [14.87] mo) were statistically similar between groups $(P=.17$ and $P=.17$, respectively). Average number of treatment sessions was 13.8 (7.3) in SV group. There were no missing patients at the final follow-up.

Preoperative DASH (HB: 27.46 [11.81] vs SV: 32.53 [16.42], $P=.22$ ), Constant (HB: 58.23 [14.23] vs SV: 54.17 [10.46], $P=.13$ ), and Rowe (HB: 51.72 [15.36] vs SV: 43.81 [19.16], $P=.12$ ) scores were statistically similar between groups (Table 1).

Postoperative DASH, Constant, and Rowe scores at sixth month were significantly improved when compared with preoperative scores in each group $(P=.001$; Table 3$)$. 
Table 3 Patients Improved Significantly After the Surgery as Shown in DASH, Constant, and Rowe Scores

\begin{tabular}{|c|c|c|c|c|}
\hline & Preoperative & $3 \mathrm{mo}$ & $6 \mathrm{mo}$ & $1 y$ \\
\hline \multicolumn{5}{|l|}{ DASH } \\
\hline Home-based group & $27.46(11.81)$ & $7.93(8.4)$ & $4.44(4.48)$ & $1.79(2.34)$ \\
\hline Supervised group & $32.53(16.42)$ & 11.08 (11.41) & $5.32(5.24)$ & $3.16(4.09)$ \\
\hline \multicolumn{5}{|l|}{ Constant } \\
\hline Home-based group & $58.23(14.23)$ & $85.29(14.02)$ & 93.27 (4.99) & $96.54(4.36)$ \\
\hline Supervised group & $54.17(10.46)$ & 85.03 (17.92) & $92.54(8.13)$ & $95.97(5.68)$ \\
\hline \multicolumn{5}{|l|}{ Rowe } \\
\hline Home-based group & $51.72(15.36)$ & 86.79 (15.64) & $95.71(4.01)$ & $98.21(2.79)$ \\
\hline Supervised group & $43.81(19.16)$ & $87.0(12.04)$ & $94.25(10.16)$ & $98.0(4.70)$ \\
\hline
\end{tabular}

Abbreviation: DASH = Disabilities of Arm Shoulder Hand.

Table 4 No Significant Difference was Observed Between Groups in Any Time Point

\begin{tabular}{lcccc}
\hline & Preoperative $^{\mathbf{a}}$ & $\mathbf{3} \mathbf{~ m o}^{\mathbf{a}}$ & $\mathbf{6} \mathbf{~ m o}^{\mathbf{a}}$ & $\mathbf{1} \mathbf{y}^{\mathbf{a}}$ \\
\hline $\begin{array}{l}\text { DASH } \\
\text { HB vs SV }\end{array}$ & 0.215 & 0.561 & 0.373 & 0.215 \\
$\begin{array}{l}\text { Constant } \\
\text { HB vs SV }\end{array}$ & 0.133 & 0.828 & 0.768 & 0.731 \\
$\begin{array}{l}\text { Rowe } \\
\text { HB vs SV }\end{array}$ & 0.120 & 0.777 & 0.815 & 0.645 \\
\hline
\end{tabular}

Abbreviations: DASH $=$ Disabilities of Arm Shoulder Hand; HB, home-based group; SV, supervised hospital-based group.

${ }^{a}$ Mann-Whitney $U$ test was used for comparison.

There was no difference in DASH, Constant, and Rowe scores at 3rd month $(P=.56, P=.83, P=.78$, respectively $)$, 6th month $(P=.37, P=.77, P=.82$, respectively $)$, and 12 th month $(P=.22$, $P=.73, P=.65$, respectively) between groups (Table 4$)$.

There were no complications in the early postoperative period. Two recurrent dislocations due to major traumatic event were observed. One patient from HB group had traumatic subluxation following a fall injury on outstretched hand at 24 months after the surgery. Another patient from the SV group had traumatic subluxation following a fall on a shoulder at 17 months after the surgery. None of the patients were reoperated and were conservatively treated. These patients were not excluded.

\section{Discussion}

Our concept of home-based rehabilitation originated from the observation that some of our patients showed poor compliance in attending our physiotherapist assisted rehabilitation protocol due to financial issues, transportation problems, or other personal reasons and still had a stable and functional shoulder at the end of 12th week. In this prospective study, we have found that there was no significant difference in clinical outcome scores of patients who were rehabilitated with home-based exercise program versus those who were rehabilitated in the physiotherapy unit, after arthroscopic anterior capsulolabral repair. Our results supported the initial hypothesis, the home-based approach providing the same results with SV therapy. The standardized rehabilitation protocol used in this study was safe and effective as a HB and SV program.

Home-based rehabilitation programs are considered to be effective in many clinical conditions. McDermott et al previously reported that the patient-controlled home-based rehabilitation protocol was safe and effective in open Bankart repair. ${ }^{15,16} \mathrm{Al}-$ though there is a vast amount of publications published on both approaches, limited comparative studies are available which report similar efficacy. ${ }^{22,23}$ Ismail and El Shorbagy ${ }^{24}$ recently compared home-based and supervised therapy programs after anterior shoulder instability surgery, in a randomized clinical trial. Similar to our data, they have also reported that a home-based program was as effective as a supervised program in increasing shoulder ROM and function after surgery.

Home-based protocols were criticized previously with low patient adherence. ${ }^{25,26}$ Essery et $\mathrm{al}^{7}$ reported that intention to engage in the home-based programs, self-motivation, self-efficacy, previous adherence to exercise-related behaviors, and social support were key factors in patient adherence. In this study, it is the patients' own decision to be enrolled in the home-based group and having a surgical intervention for recovery facilitates self-motivation and self-efficacy in patients. We believe that these factors provide high adherence to home-based protocols when efficient protocols are available.

Recent studies on the role of mechanobiology in soft tissue healing revealed that controlled loading can enhance healing..$^{27,28}$ On the other hand, immobilization in the sling creates a time window for soft tissue healing. Therefore, a fine balance is critical to achieve soft tissue healing while improving ROM. ${ }^{27,29}$ Immobilization period after open Bankart repair ranges from 2 days to 5 weeks in the literature. ${ }^{1,16,18}$ We preferred 4 weeks of immobilization, as in similar studies. Patients were not allowed to perform external rotation at abduction. ${ }^{1,4}$ American Society of Shoulder and Elbow Therapist consensus guideline also suggests avoiding excessive stress in the first 12 weeks, where strength of the repair is solely based on the anchor fixation. Sling immobilization with shoulder ROM less than $30^{\circ}$ was recommended. ${ }^{30}$ We have also allowed all table top activities without sling, during the immobilization period. It was shown that absolute immobilization has no beneficial effects on healing and currently is not recommended after shoulder immobilization surgery. ${ }^{15}$

In a randomized controlled trial, an accelerated rehabilitation program was assessed in a selected patient group. The authors reported that early mobilization of the operated shoulder after arthroscopic Bankart repair does not increase the recurrence rate; accelerated rehabilitation program with passive ROM exercises at the first postoperative day promoted functional recovery, reducing postoperative pain, and allowing patients an early return to desired activities. ${ }^{15}$ In this study, we have shown that a home-based program is also safe regarding the recurrence and is effective in 
clinical scores. From these 2 studies, it can be concluded that accelerated home-based rehabilitation protocols can be tested in selected patients, against SV accelerated protocols.

Hospital-based rehabilitation allows therapists to follow detailed and structured programs, which were thought to be more efficient compared with conventional care. For that reason, American Society of Shoulder and Elbow Therapist guidelines were constructed in a very detailed manner to facilitate clinical outcomes. However, it was shown that detailed and structured rehabilitation programs have no superiority over conventional standard care. ${ }^{8}$ This study also supported their results, stating that a strict supervision was not necessary as patients showed similar outcomes.

In our series, we have observed recurrent dislocation in 2 patients (one from each group), which was related to significant traumatic event. All other patients remained stable at the end of the first year. Therefore, it can be concluded that HB and SV rehabilitation protocols are both safe. In addition, patient selection was another key factor to achieve these results. Results following revision Bankart surgery, posterior instability, glenoid bone loss, and patients with specific comorbidities may differ from our cohort.

In this study, the method for patient selection can be considered as a limitation. Although a nonrandomized allocation was preferred, there was no significant difference between the 2 groups. Patient education has a paramount importance in the success of home-based therapy programs. Well-educated and motivated patients may perform better with home-based programs. We did not utilize any outcome measures to reveal the effects of education and motivation on the programs. Randomized studies comparing $\mathrm{HB}$ and SV rehabilitation approaches may overcome this limitation. Also, accelerated home-based protocols can be tested against conventional approaches.

\section{Conclusion}

A home-based, patient-controlled rehabilitation program following 4 weeks of immobilization is a safe and effective method for the rehabilitation of patients after arthroscopic Bankart repair. Meticulous patient selection and surgical technique allowing optimal capsular shift and tensioning is still the most important factor affecting patient outcomes.

\section{References}

1. Cole BJ, L'Insalata J, Irrgang J, Warner JJ. Comparison of arthroscopic and open anterior shoulder stabilization. A two to six-year follow-up study. J Bone Joint Surg Am. 2000;82-A(8):1108-1114. PubMed ID: 10954100 doi:10.2106/00004623-200008000-00007

2. Kim SH, Ha KI, Kim SH. Bankart repair in traumatic anterior shoulder instability: open versus arthroscopic technique. Arthroscopy. 2002;18(7):755-763. PubMed ID: 12209434

3. Bottoni CR, Smith EL, Berkowitz MJ, Towle RB, Moore JH. Arthroscopic versus open shoulder stabilization for recurrent anterior instability: a prospective randomized clinical trial. Am J Sports Med. 2006;34(11):1730-1737. PubMed ID: 16735589 doi:10.1177/ 0363546506288239

4. Gartsman GM, Roddey TS, Hammerman SM. Arthroscopic treatment of anterior-inferior glenohumeral instability. Two to five-year followup. J Bone Joint Surg Am. 2000;82-A(7):991-1003. PubMed ID: 10901314

5. Boileau P, Villalba M, Hery JY, Balg F, Ahrens P, Neyton L. Risk factors for recurrence of shoulder instability after arthroscopic
Bankart repair. J Bone Joint Surg Am. 2006;88(8):1755-1763. PubMed ID: 16882898 doi:10.2106/JBJS.E.00817

6. Ma R, Brimmo OA, Li X, Colbert L. Current concepts in rehabilitation for traumatic anterior shoulder instability. Curr Rev Musculoskelet Med. 2017;10(4):499-506. PubMed ID: 29038953 doi:10.1007/ s12178-017-9449-9

7. Essery R, Geraghty AW, Kirby S, Yardley L. Predictors of adherence to home-based physical therapies: a systematic review. Disabil Rehabil. 2017;39(6):519-534. PubMed ID: 27097761 doi:10.3109/ 09638288.2016.1153160

8. Damkjaer L, Petersen T, Juul-Kristensen B. Is the American Society of Shoulder and Elbow Therapists' rehabilitation guideline better than standard care when applied to Bankart-operated patients? A controlled study. Clin Rehabil. 2015;29(2):154-164. PubMed ID: 24994769 doi:10.1177/0269215514539819

9. Hayden JA, van Tulder MW, Tomlinson G. Systematic review: strategies for using exercise therapy to improve outcomes in chronic low back pain. Ann Intern Med. 2005;142(9):776-785. PubMed ID: 15867410

10. Vermeire E, Hearnshaw H, Van Royen P, Denekens J. Patient adherence to treatment: three decades of research. A comprehensive review. J Clin Pharm Ther. 2001;26(5):331-342. PubMed ID: 11679023

11. Borello-France D, Burgio KL, Goode PS, et al. Adherence to behavioral interventions for urge incontinence when combined with drug therapy: adherence rates, barriers, and predictors. Phys Ther. 2010;90(10):1493-1505. PubMed ID: 20671098 doi:10.2522/ptj. 20080387

12. Alexandre NM, Nordin M, Hiebert R, Campello M. Predictors of compliance with short-term treatment among patients with back pain. Rev Panam Salud Publica. 2002;12(2):86-94. PubMed ID: 12243693

13. Green MR, Christensen KP. Arthroscopic versus open Bankart procedures: a comparison of early morbidity and complications. Arthroscopy. 1993;9(4):371-374. PubMed ID: 8216566

14. Morgan CD, Bodenstab AB. Arthroscopic Bankart suture repair: technique and early results. Arthroscopy. 1987;3(2):111-122. PubMed ID: 3300681

15. Kim SH, Ha KI, Jung MW, Lim MS, Kim YM, Park JH. Accelerated rehabilitation after arthroscopic Bankart repair for selected cases: a prospective randomized clinical study. Arthroscopy. 2003;19(7): 722-731. PubMed ID: 12966380

16. McDermott DM, Neumann L, Frostick SP, Wallace WA. Early results of Bankart repair with a patient-controlled rehabilitation program. J Shoulder Elbow Surg. 1999;8(2):146-150. PubMed ID: 10226967

17. Solway S, Beaton D, McConnell S, Bombardier C. The DASH Outcome Measure User's Manual. 2nd ed. Toronto, Canada: Institute for Work \& Health; 2002.

18. Rowe CR, Patel D, Southmayd WW. The Bankart procedure: a longterm end-result study. J Bone Joint Surg Am 1978;60(1):1-16. PubMed ID: 624747

19. Celik D. Turkish version of the modified Constant-Murley score and standardized test protocol: reliability and validity. Acta Orthop Traumatol Turc. 2016;50(1):69-75. PubMed ID: 26854052 doi:10. 3944/aott.2016.14.0354

20. Constant CR, Gerber C, Emery RJ, Sojbjerg JO, Gohlke F, Boileau P. A review of the Constant score: modifications and guidelines for its use. J Shoulder Elbow Surg. 2008;17(2):355-361. PubMed ID: 18218327 doi:10.1016/j.jse.2007.06.022

21. Constant CR, Murley AH. A clinical method of functional assessment of the shoulder. Clin Orthop Relat Res. 1987;(214):160-164.

22. Florez-Garcia M, Garcia-Perez F, Curbelo R, et al. Efficacy and safety of home-based exercises versus individualized supervised outpatient 
physical therapy programs after total knee arthroplasty: a systematic review and meta-analysis. Knee Surg Sports Traumatol Arthrosc. 2017;25(11):3340-3353. PubMed ID: 27401004 doi:10.1007/ s00167-016-4231-x

23. Su TL, Chen AN, Leong CP, et al. The effect of home-based program and outpatient physical therapy in patients with head and neck cancer: a randomized, controlled trial. Oral Oncol. 2017;74: 130-134. PubMed ID: 29103741 doi:10.1016/j.oraloncology.2017. 10.002

24. Ismail MM, El Shorbagy KM. Motions and functional performance after supervised physical therapy program versus homebased program after arthroscopic anterior shoulder stabilization: a randomized clinical trial. Ann Phys Rehabil Med. 2014;57(6-7): 353-372. PubMed ID: 25164471 doi:10.1016/j.rehab.2014. 06.002

25. Campbell R, Evans M, Tucker M, Quilty B, Dieppe P, Donovan JL. Why don't patients do their exercises? Understanding noncompliance with physiotherapy in patients with osteoarthritis of the knee. J Epidemiol Community Health. 2001;55(2):132-138. PubMed ID: 11154253 doi:10.1136/jech.55.2.132
26. Sluijs EM, Kok GJ, van der Zee J. Correlates of exercise compliance in physical therapy. Phys Ther. 1993;73(11):771-782. discussion 783-776. PubMed ID: 8234458 doi:10.1093/ptj/73.11.771

27. Killian ML, Cavinatto L, Galatz LM, Thomopoulos S. The role of mechanobiology in tendon healing. J Shoulder Elbow Surg. 2012; 21(2):228-237. PubMed ID: 22244066 doi:10.1016/j.jse.2011. 11.002

28. Cuff DJ, Pupello DR. Prospective randomized study of arthroscopic rotator cuff repair using an early versus delayed postoperative physical therapy protocol. J Shoulder Elbow Surg. 2012;21(11): 1450-1455. PubMed ID: 22554876

29. Galatz LM, Charlton N, Das R, Kim HM, Havlioglu N, Thomopoulos S. Complete removal of load is detrimental to rotator cuff healing. $J$ Shoulder Elbow Surg. 2009;18(5):669-675. PubMed ID: 19427237 doi:10.1016/j.jse.2009.02.016

30. Gaunt BW, Shaffer MA, Sauers EL, Michener LA, McCluskey GM, Thigpen C. The American Society of Shoulder and Elbow Therapists' consensus rehabilitation guideline for arthroscopic anterior capsulolabral repair of the shoulder. J Orthop Sports Phys Ther. 2010;40(3): 155-168. PubMed ID: 20195022 doi:10.2519/jospt.2010.3186 